\title{
Aquarius Whole Range Calibration: Celestial Sky, Ocean, and Land Targets
}

\author{
Emmanuel P. Dinnat \\ Chapman University \\ Cryospheric Sciences Laboratory, NASA-GSFC \\ Greenbelt, MD, U.S.A. \\ emmanuel.dinnat@nasa.gov \\ David M. Le Vine \\ Cryospheric Sciences Laboratory \\ NASA-Goddard Space Flight Center \\ Greenbelt, MD, U.S.A.
}

\author{
Rajat Bindlish \\ USDA-ARS \\ Beltsville, MD, U.S.A. \\ Jeffrey R. Piepmeier \\ Microwave Instrument Technology Branch \\ NASA-Goddard Space Flight Center \\ Greenbelt, MD, U.S.A.
}

\author{
Shannon T. Brown \\ NASA- Jet Propulsion Laboratory \\ Pasadena, CA, U.S.A.
}

\begin{abstract}
Aquarius is a spaceborne instrument that uses Lband radiometers to monitor sea surface salinity globally. Other applications of its data over land and the cryosphere are being developed. Combining its measurements with existing and upcoming L-band sensors will allow for long term studies. For that purpose, the radiometers calibration is critical. Aquarius measurements are currently calibrated over the oceans. They have been found too cold at the low end (celestial sky) of the brightness temperature scale, and too warm at the warm end (land and ice). We assess the impact of the antenna pattern model on the biases and propose a correction. We re-calibrate Aquarius measurements using the corrected antenna pattern and measurements over the Sky and oceans. The performances of the new calibration are evaluated using measurements over well instrument land sites.
\end{abstract}

Keywords-Microwave; Radiometry; L-band; Aquarius; Calibration

\section{INTRODUCTION}

Aquarius is a NASA spaceborne instrument that combines three L-band radiometers and one scatterometer at L-band. It is onboard the SAC-D spacecraft by the Argentine space agency Comisión Nacional de Actividades Espaciales (CONAE) [1]. Its scientific objective is to produce global maps of sea surface salinity (SSS) with a monthly precision of $0.2 \mathrm{psu}$ at $150 \mathrm{~km}$ ground resolution. The radiometers are the prime instrument to measure SSS. The scatterometer is used to help with the correction of the impacts of sea surface roughness on the radiometric signal [2]. The spacecraft is on a sun-synchronous polar orbit with ascending equatorial crossing node at $6 \mathrm{pm}$. Aquarius radiometers use three feed horns and a reflector (time shared with the scatterometer) to project three beams across the orbital plane sampling the Earth surface in a push-broom fashion.

This research was supported by the NASA grant \# NNX10AV23G.

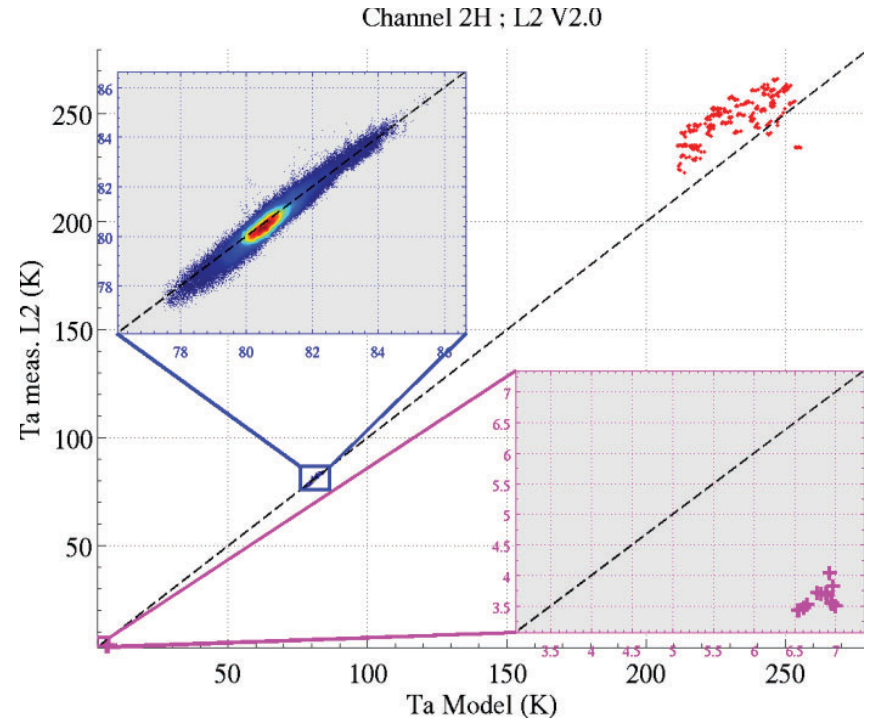

Fig. 1. $T_{a}$ measured by Aquarius versus simulated over (magenta) the Celestial Sky, (blue) ocean and (red) land, horizontal polarization of the middle beam. Insets are close-ups of (top) ocean and (right) Sky data.

Aquarius/SAC-D was launched on June 10, 2011. It is the second spaceborne mission to monitor the Earth using L-band radiometry after the launch of ESA's Soil Moisture and Ocean Salinity (SMOS) in late 2009 (first available data at beginning of 2010) [3]. Coming after both these missions will be NASA's Soil Moisture Active-Passive (SMAP) in 2014 [4], and likely a follow-on mission for SMOS toward the end of the decade. With SMAP's end of life planned for 2018 at the earliest, this makes the L-band continuity extends over about a decade. In order to use L-band data from these various instruments for 

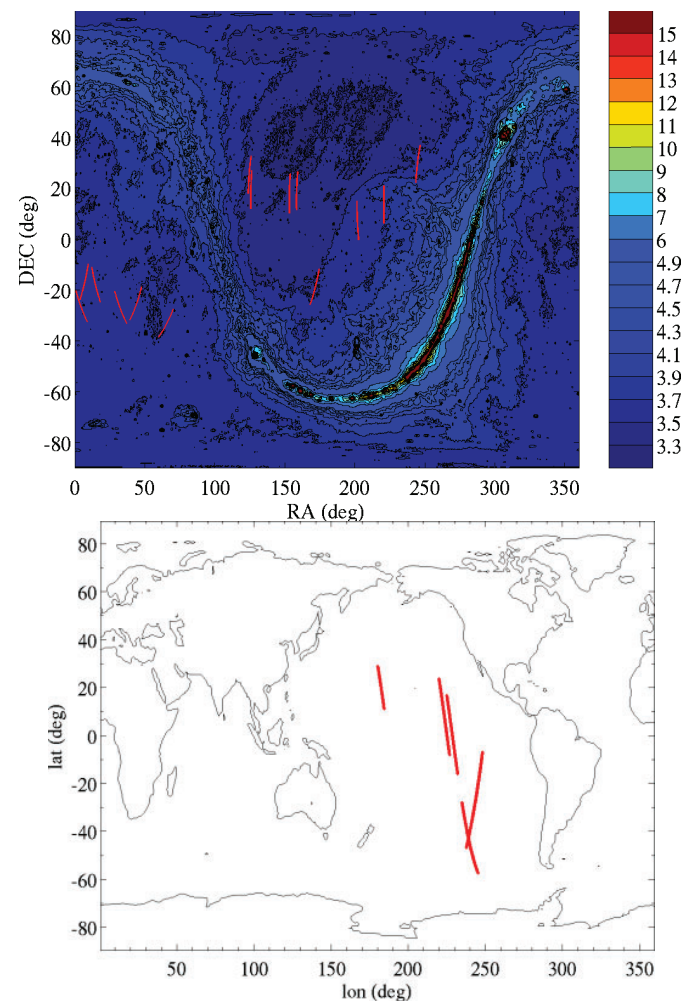

Fig. 2. (top) tracks of the middle beam over the Sky Tb map during the 14 CSC maneuvers between March 2012 and March 2014. (bottom) Sub-satellite track locations during the maneuvers.

long term scientific studies of oceans and the cryosphere [5], calibration will be of critical importance.

Aquarius uses noise diode to perform internal calibration. This provides a good temporal stability but the uncertainty in the absolute temperature of the noise diode result in a bias in antenna temperature $\left(T_{a}\right)$ of up to a few Kelvin. In order to correct for such biases, Aquarius measurements are compared to forward radiative transfer simulations over Open Ocean using an ocean model to provide SSS [6]. The difference between measurements and simulation averaged globally over a few days is subtracted from the measurements [7]. Because the same model is used for the forward radiative transfer and the retrieval algorithm, the process is self-consistent and results in removing a global bias between retrieved SSS and the ancillary SSS used for the calibration.

As part of the vicarious calibration approach, the spacecraft is rotated upside down about once a month in order to observe the Celestial Sky which has a brightness temperature of the order of $3.5 \mathrm{~K}$. Comparisons with simulation have showed that the measured $T_{a}$ are too cold by about $2.5 \mathrm{~K}$ (Fig. 1). At the warm end of the temperature scale, measured $T_{a}$ over land are too warm by $5-10 \mathrm{~K}$ (Fig. 1). We present a study to identify the reason for such discrepancies and propose ways to correct for it. In Section II, we present the nominal calibration algorithm used for Aquarius over oceans (version 2.0 of the data) and the method for assessing its performances over the Sky (cold target) and land (warm target). In Section III, we compute a correction for the radiometer's antenna pattern model (i.e. create a hybrid model) to mitigate biases observed. In Section

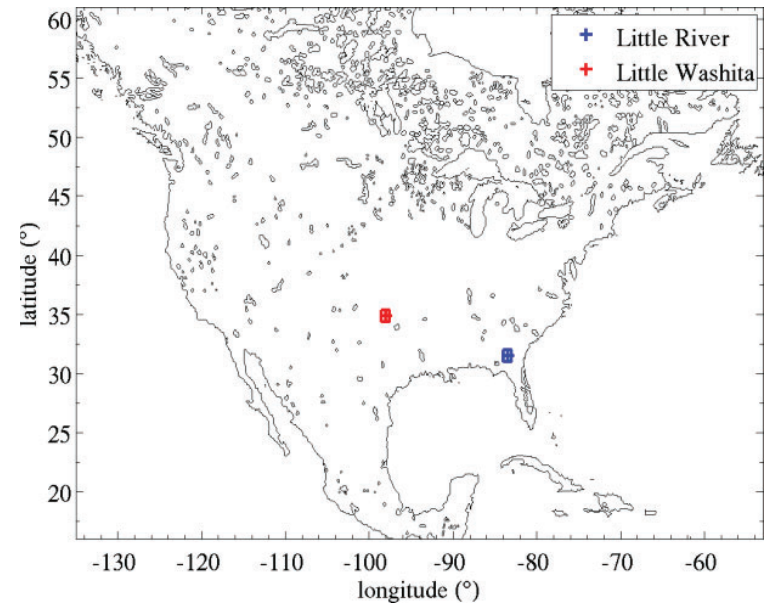

Fig. 3. Map of the location of the land validation sites. These are the Little River and Little Washita watershed site monitored by the USDA with in situ measurements of soil temperature and moisture.

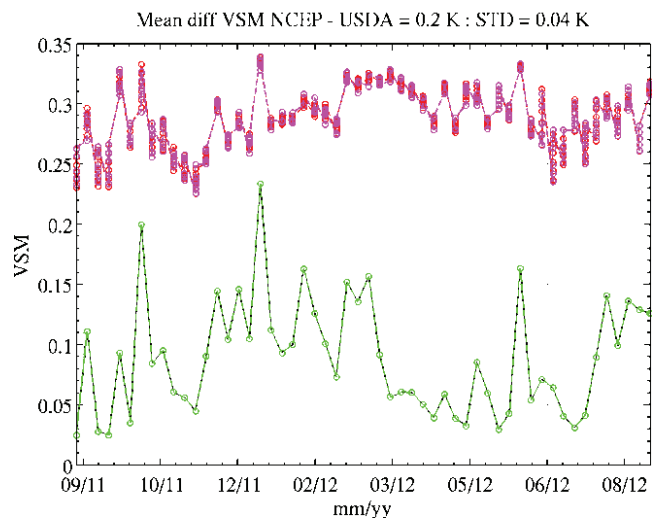

Fig. 4. Comparison of the volumetric soil moisture at the Little River watershed USDA site (green) measured in situ and (magenta) as predicted by NCEP.

IV, we assess the whole range calibration over the Sky, oceans and land using the hybrid pattern model. We summarize the results in Section V.

\section{ViCARIOUS CALIBRATION ALGORITHM AND VALIDATION OVER SKY AND LAND}

\section{A. The Aquarius Simulator}

The Aquarius simulator is a forward radiative transfer model that computes the antenna temperature for the three Aquarius radiometers [8]. The contributions from all around the antenna are weighted and integrated over the antenna gain patterns $(G)$ and the antenna temperature $T_{a}$ writes

$$
\begin{gathered}
T_{a}=1 / 4 \pi \int T_{b} G \mathrm{~d} \Omega \\
=1 / 4 \pi \int_{\text {earth }} T_{b} G \mathrm{~d} \Omega+1 / 4 \pi \int_{\text {sky }} T_{b} G \mathrm{~d} \Omega
\end{gathered}
$$

The contributions from the Sky include the galaxy and cosmic background emission, the Sun and the Moon. The 


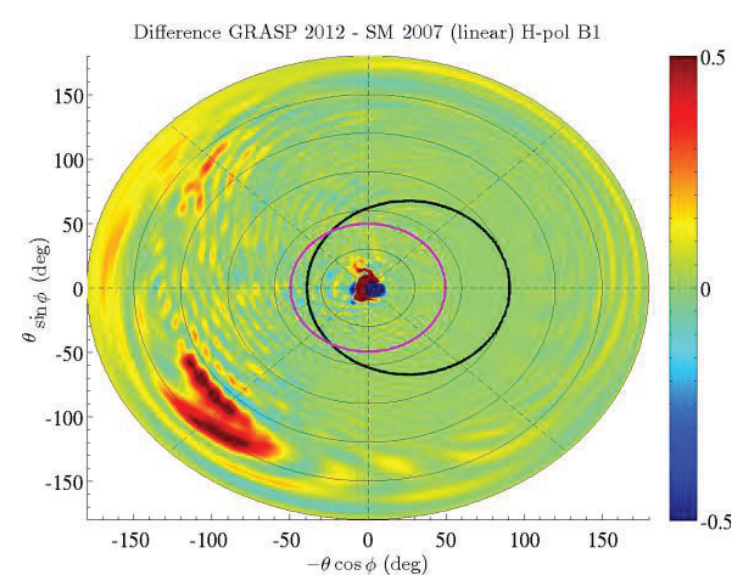

Fig. 5. Difference between the antenna gain pattern models derived from GRASP and from a scale model of the spacecraft and instrument for the horizontal polarization of the inner beam. The black circle reports the edge of the Earth in nominal (i.e. Earth) pointing. The magenta circle reports the limit beyond which the gain of the GRASP model is normalized to that of the scale model to create the hybrid model (see text).

contributions from the Earth include emission from oceans, land and ice present in the field of view, atmospheric contributions (i.e. upward emission, attenuation of surface emission and contributions reflected at the surface such as downward atmospheric emission) and Faraday rotation that results in polarizations mixing. At some locations over the oceans, there are also contributions reflected at the surface such as the emission from the Sky, the Sun [9] and Moon [10].

\section{B. Celestial Sky Calibration Maneuvers}

The Celestial Sky is an important calibration target because it offers large regions of homogeneous brightness temperature $T_{b}$ (changes less than $0.2 \mathrm{~K}$ ), is stable in time and relatively well known. During a Celestial Sky Calibration (CSC) maneuver, the spacecraft is pitched by $180^{\circ}$ for a 5 minutes period in order for the radiometers to point to the Sky and have most of the antenna power $(\sim 97 \%)$ off the Earth surface. Because there are still a few percent of power coming from the Earth, the CSC maneuvers are performed over oceans in order to minimize the sources of errors (radio frequency interferences and surface emission uncertainty). Between March 2012 and March 2014, 14 maneuvers have been conducted with the beams pointing toward calm region of the Sky and the spacecraft flying over the open ocean (Fig. 2).

\section{Land Validation Sites}

The comparisons over land are performed at sites monitored by the USDA. We use the Little River and Little Washita watersheds (Fig. 3) that are collocated with Aquarius middle and outer beam ground tracks respectively. The in situ soil moisture (Fig. 4) shows significant differences with the value derived from NCEP model that is used nominally in the Aquarius processing. This demonstrates the importance of performing land validations where the knowledge of environmental parameters is optimum.

\section{IMPROVING THE GAIN PATtern MODEL WiTH THE HYBRID MODEL}

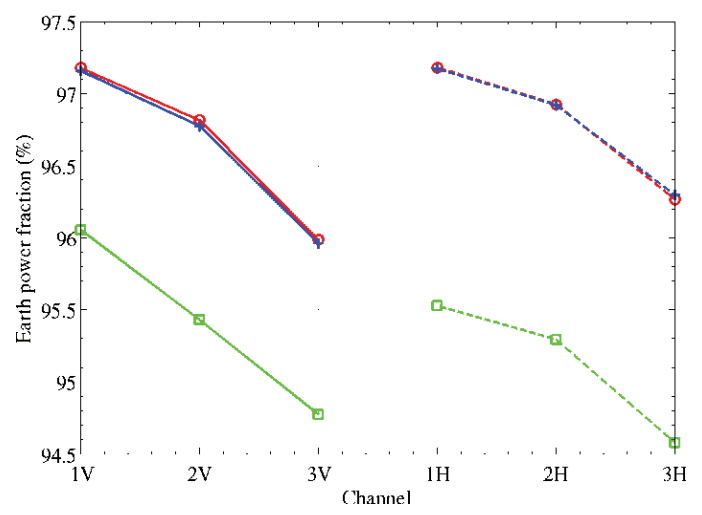

Fig. 6. Fraction (in \%) of antenna gain power over the Earth for the six Aquarius channel (beams $1-3$ and vertical and horizontal polarizations). The pattern are from (red) the scale model SM, (green) the GRASP numerical simulations G12, and (blue) and the Hybrid model.

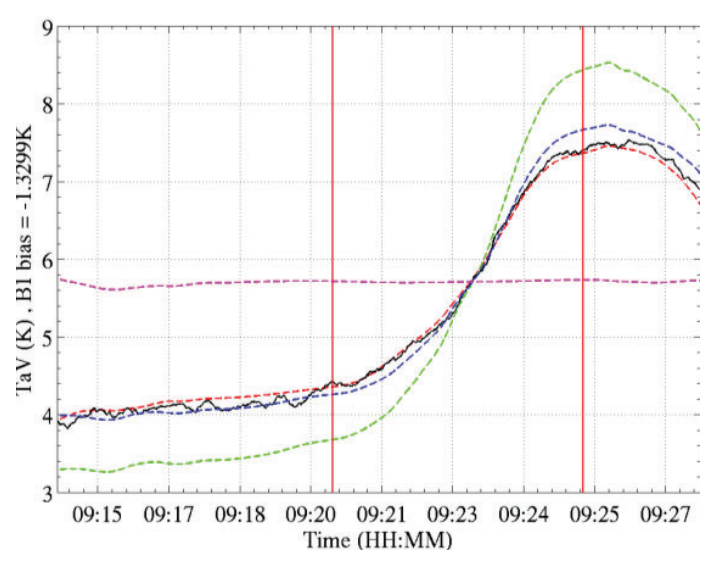

Fig. 7. Antenna temperature for the vertical polarization of the inner beam during the ocean/land crossing celestial sky calibration maneuver. The curves are (black)Aquarius measurements and simulations using (red) the scale model, (green) the GRASP model and (blue) the hybrid model for the antenna gain patterns. The magenta dashed line is the simulated Sky Tb.

A critical component of the forward model used to calibrate and validate the radiometer measurements is the antenna gain pattern (see (1)). At the beginning of the Aquarius mission (pre version 2.0 of the data), antenna patterns derived from a scale model (SM) of the spacecraft and instrument were used. In 2012, a newer model derived from numerical simulation became available and was used in V2.0 of the data processing. The model was derived from the Generalized Reflector Antenna analysis Software Package (GRASP). A few important differences were found between both models, among which GRASP showed lower cross-polarization gain and a higher spillover ratio. The latter difference is illustrated in Fig. 5 , with the bright red area around $\mathrm{x}=\mathrm{y}=-100^{\circ}$ showing the relative large difference between both patterns in the spillover region.

The fraction of integrated power over the Earth for both sets of antenna patterns is reported in Fig. 6. The GRASP model shows Earth power fraction systematically lower by 1 $2 \%$ for all channels. This difference results in differences over the ocean $(\mathrm{Tb} \sim 100 \mathrm{~K})$ of the order of a Kelvin, and about twice that difference over land. 


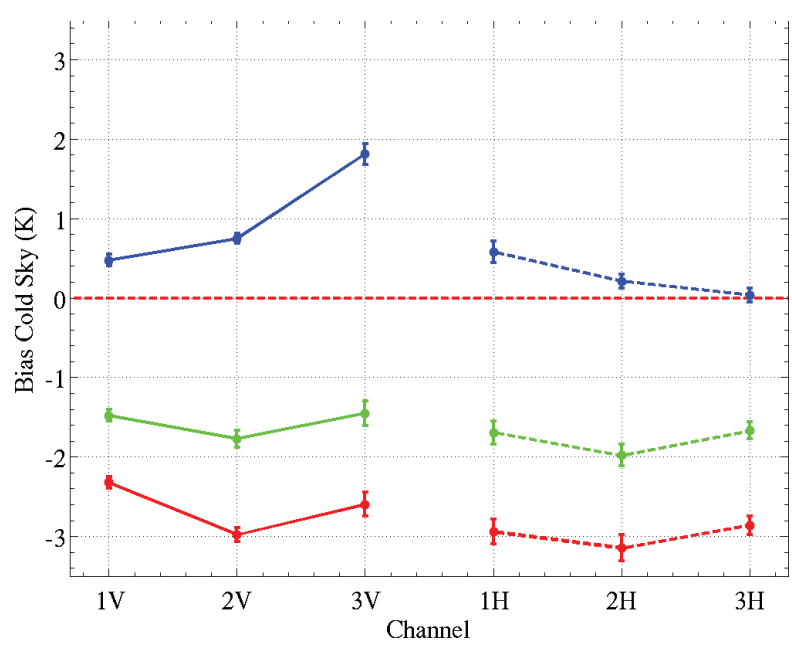

Fig. 8. Biases computed as the difference between the antenna temperatures measured by Aquarius minus simulations over the celestial sky during the calibration maneuvers for six Aquarius channels. The points represent the median bias and the error bars report the standard deviation for 14 maneuvers spread over 2 years. The simulations use (red) GRASP or (green, blue) the hybrid model for the antenna patterns. They are compared to (red, green) the V2.0 or (blue) pre-release of V3.0 of the Aquarius data

A dedicated celestial Sky maneuver was carried out over a transition ocean/land in order to determine the best model for the spillover (Fig. 7). The simulation of Sky Tb alone predicts a constant contribution over the calibration period. This means that the large $(\sim 3 \mathrm{~K})$ increase in measured $\mathrm{Ta}$ is due to the Earth contribution and is largely dependent on the spillover. The simulation using the GRASP model overestimates the increase by about $2 \mathrm{~K}$ and the SM reproduces the observed increase much more accurately (within $0.1 \mathrm{~K}$ of the data). This shows that the GRASP model has too large of a spillover, namely in this configuration has too much antenna power over the Earth.

Because the GRASP model has showed better performances in accounting for the cross-polarization gain and boresight alignment, it is desirable to keep some of its features while avoiding the inaccurate spillover. For that purpose, a hybrid model was developed using the main features of the GRASP model but adjusting its front to back power ratio. Namely, the total gain of the GRASP model integrated for polar angles larger than $50^{\circ}$ off the boresight was normalized to that of the scale model, in effect reducing the intensity of the spillover in the GRAPS model. The Earth power fraction for the hybrid model is reported in Fig. 6. It matches closely the scale model, within about $0.1 \%$. The blue curve in Fig. 7 shows that the simulations using the hybrid model match the performance of the scale model in reproducing the measurements of the ocean/land CSC, confirming that the spillover adjustment is performing as intended.

\section{Whole RANGe CALIBRATION USING THE Hybrid GAIN PATTERN}

A new set antenna temperatures simulations are computed over the sky, ocean, and land using the hybrid gain pattern described in Section III. Fig. 8 reports the improvement at the cold end (celestial sky) bias. The initial bias for V2.0 of the data (red curves) was around $-2.5 \mathrm{~K}$ for all channels. Using a
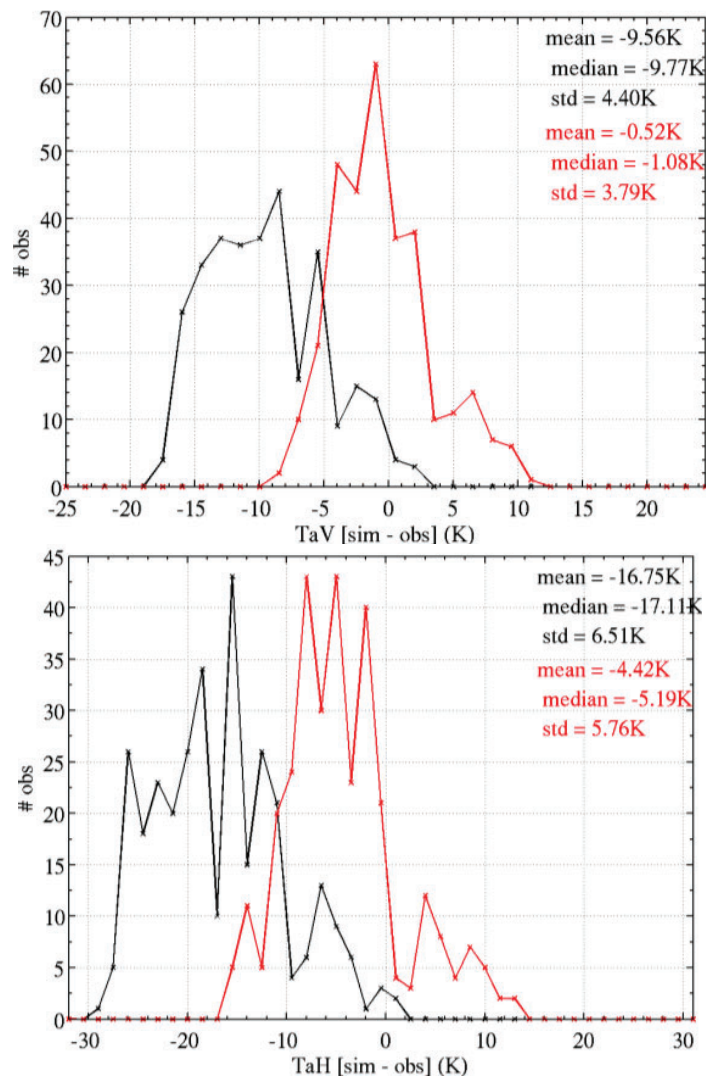

Fig. 9. Distribution of the differences between $T_{a}$ simulated and measured over the Little River land validation site for (black) V2.0 versus GRASP pattern and NCEP ancillary data and (red) recalibrated data versus hybrid pattern and in situ ancillary data. This is for the horizontal polarizastion of the middle beam.

more realistic antenna pattern for the simulations (i.e. the hybrid model) decreased the biases by about $1 \mathrm{~K}$ (green curves). However, the simulations are still being compared to data calibrated over the ocean using the GRASP model. The blue curve reports comparisons of simulations with a prerelease of V3.0 of the Aquarius data that include a correction for the spillover ratio very similar to that described here. The bias has been significantly reduced $(0.7 \mathrm{~K}$ or less $)$ for all channels, except the vertical polarization of the outer beam $(3 \mathrm{~V})$. The causes for this discrepancy are still under investigation.

To recalibrate the data over the whole range of $T_{a}$, a linear regression is performed between the Aquarius data and the simulation over the Sky and ocean. The results are checked over reference land sites with a well instrumented network of in situ measurements of environmental parameters (soil moisture and temperature). The new recalibrated data are computed as

$$
T_{a, \text { new }}=a \cdot T_{a, L 2}+b
$$

with $a$ and $b$ computed such that

$$
T_{a, \text { new }}=T_{a, \text { sim }}
$$


where $T_{a, s i m}$ are antenna temperatures simulated using the hybrid gain pattern.

The improvement of the new calibration and the use of in situ data compared to V2.0 of the data is reported in Fig. 9 for the Little River watershed. The bias is reduced by a few Kelvins as well as the scatter between the model and measurements. About half of the reduction in bias in due to the use of better ancillary data for soil moisture and temperature (in situ vs NCEP which is too wet) and the other half is due to the hybrid pattern that corrects for the inaccuracies in spillover. The Little Washita watershed (not shown) shows also a significant reduction in bias and scatter, but the remaining scatter is still significant due to model deficiencies (some large fluctuations in $T_{a}$ correlated with soil moisture changes are not accurately captured by the model). Despite the uncertainty in the model that prevents validation at the sub-Kelvin scale as is possible over the Sky and ocean, land comparisons clearly show a significant improvement due to the new calibration approach.

\section{CONCLUSIONS}

As part of the nominal processing, Aquarius antenna temperatures are calibrated empirically to radiative transfer simulations over oceans, removing a global average bias with respect to the reference ancillary field for SSS. The spacecraft is also rotated upside down monthly in order for the instrument to observe the celestial Sky. It is found that the Sky measurements are biased by about $-2.5 \mathrm{~K}$. At the warm end of the measurements, a bias of the order of $5-10 \mathrm{~K}$ is observed over land.

The comparison of two different models for the antenna gain patterns showed significant differences in the intensity of the spillover. A dedicated celestial Sky maneuver was carried out over a transition ocean/land in order to determine the best model for the spillover. From these results, a hybrid antenna gain pattern was developed, combining the strengths of both models.

The forward model simulations for the Sky, ocean and land were re-computed using the hybrid patterns and compared to a pre-release of Aquarius data V3.0 that include a correction for the spillover ratio. The bias over the Sky was significantly reduced for all channels $(0.7 \mathrm{~K}$ or less $)$ except for the $\mathrm{V}$-pol of the outer beam that went from a $-2.6 \mathrm{~K}$ to $\mathrm{a}+1.8 \mathrm{~K}$ bias.

The residual bias was corrected using a linear regression between the measured and simulated Ta over the Sky and the ocean. The performances were evaluated over well instrumented land sites for the middle and outer beams. The biases and scatter of the data versus the simulations were significantly reduced. The improvements of the new calibration are clear over the whole range of antenna temperatures.

\section{REFERENCES}

[1] D. Le Vine, G. S. E. Lagerloef, and S. Torrusio, "Aquarius and remote sensing of sea surface salinity from space," Proceedings of the IEEE, vol. 98, no. 5, pp. 688-703, May 2010.

[2] S. Yueh, et al., "L-band passive and active microwave geophysical model functions of ocean surface winds and applications to Aquarius retrieval," IEEE Trans. Geosci. Remote Sensing, vol. 51, no. 9, pp. 4619-4632, Sept 2013.

[3] Y. Kerr, et al., "The SMOS mission: New tool for monitoring key elements ofthe global water cycle," Proceedings of the IEEE, vol. 98, no. 5, pp. 666-687, May 2010.

[4] D. Entekhabi, et al., "The Soil Moisture Active Passive (SMAP) mission," Proceedings of the IEEE, vol. 98, no. 5, pp. 704-716, May 2010.

[5] L. Brucker, E. P. Dinnat, and L. Koenig, "Weekly-gridded Aquarius Lband radiometer/scatterometer observations and salinity retrievals over the polar regions, Part 2: Initial product analysis," The Cryosphere, in press, 2014.

[6] E. P. Chassignet et al., "The HYCOM (HYbrid Coordinate Ocean Model) data assimilative system," Journal of Marine Systems, vol. 65, Issues 1-4, pp. 60-83, March 2007.

[7] J. Piepmeier, et al., "Aquarius radiometer post-launch calibration for product version 2," NASA, Tech. Rep. AQ-014-PS-0015, 2013.

[8] D. M. Le Vine, E. P. Dinnat, , S. Abraham, P. de Matthaeis, and F. J. Wentz, "The Aquarius simulator and cold-sky calibration," IEEE Trans. Geosci. Remote Sensing, vol. 49, no. 9, pp. 3198-3210, September 2011.

[9] E. P. Dinnat and D. M. Le Vine, "Impact of sun glint on salinity remote sensing: An example with the Aquarius radiometer," IEEE Trans. Geosci. Remote Sensing, vol. 46, no. 10, pp. 3137-3150, October 2008.

[10] E. P. Dinnat, S. Abraham, D. M. Le Vine, P. de Matthaeis, and D. Jacob, "Effect of emission from the Moon on remote sensing of sea surface salinity: an example with the Aquarius radiometer." IEEE Geoscience and Remote Sensing Letters, vol. 6, no. 2, pp. 239-243, April 2009. 